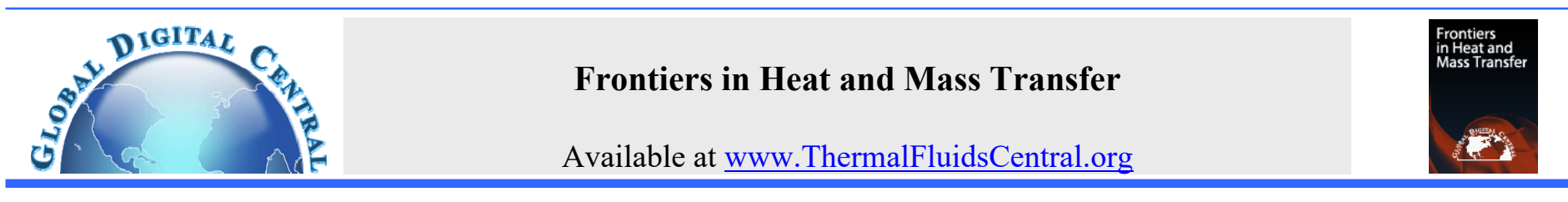

\title{
THERMAL EFFECTS IN BINGHAM PLASTIC FLUID FILM LUBRICATION OF ASYMMETRIC ROLLERS
}

\author{
Revathi Gadamsettya, ${ }^{\mathrm{a}}$ Venkata Subrahmanyam Sajja ${ }^{\mathrm{b}}$, P. Sudam Sekhar ${ }^{\mathrm{c}}$, Dhaneshwar Prasad ${ }^{\mathrm{d}, \dagger}$ \\ ${ }^{a}$ Research Scholar, Department of Mathematics, Koneru Lakshmaiah Education Foundation, Guntur-522502, Andhra Pradesh, India \\ ${ }^{b}$ Department of Mathematics, Koneru Lakshmaiah Education Foundation, Guntur-522502, Andhra Pradesh, India \\ ${ }^{c}$ Division of Mathematics, Vignan's Foundation for Science, Technology and Research, Guntur-522231, Andhra Pradesh, India \\ ${ }^{d}$ Department of Mathematics, Kanchi Mamunivar Centre for Post Graduate Studies, Puducherry-605008, India
}

\begin{abstract}
Hydrodynamic lubrication characteristics of asymmetric rollers lubricated by non-Newtonian incompressible Bingham plastic fluid are analyzed in this work. It narrates the qualitative research with the rigid system in which the viscosity of the particular non-Newtonian Bingham plastic substance is considered to become the function of hydrodynamic pressure. The equations considered in this work like equation of motion along with continuity and energy equations are solved numerically using MATLAB after particular analytical steps. Resulting from this particular work, it is identified that there is some notable change in temperatures, pressure, load and traction forces with Newtonian and also non-Newtonian fluids both. Moreover, the results acquired in this work have attained good agreement with earlier published work.
\end{abstract}

Keywords: Hydrodynamic lubrication, Non-Newtonian, Bingham plastic, Thermal effects, Viscosity, Incompressible.

\section{INTRODUCTION}

The traditional hypothesis of hydrodynamic lubricant certainly expects that the fluid acts basically as a Newtonian gooey liquid. NonNewtonian conduct is, in any case, perpetually saw in different lubricant forms. This might be an outcome of extreme operational prerequisites or might be because of the way that liquids, for example, liquid plastics, pulps, slurries, emulsions, oils, and so on are being created mechanically in expanding amounts and discovering use in lubricant streams. Such liquids abuses the Newtonian propose which accept a straight connection between shear pressure and pace of shear. Different hypotheses have been hypothesized as of late to portray the stream conduct of non-Newtonian liquids. (Prawal Sinha and Chandan Singh, 1982) Assumptive studies of the fluid using Bingham model date back to Milne (Milne, 1954). He examined the very simple one dimensional slider and journal bearings, in addition to concluded that rigid 'cores' may be attached to be able to one or another surfaces. The Bingham plastic type of non-Newtonian fluid stream behavior was employed to narrate the lubricants behavior (Christopher and Tichy, 1992).

The hydrodynamic bearing is a type of machine segment utilized so as to transmit a load between two parts of mechanical approach moving in relative action. In this sort of related bearing, lubricating film separates the moving bearing surfaces. In many bearing devices, lubricants are used which the non-Newtonian actions are reasonably model applying Bingham plastic material which generally joins a good conduct with shear stress esteems under a basic yield stress, with constant viscosity over that yield stress (Stefan, 2020). Vola et al. (2003) suggested a method to compute unsteady flow of Bingham fluids without regularizing the constitutive law. Mitsoulis and Matsoukas (2005) presented some effects with squeeze flow associated with the Bingham plastics showing the state of the free surface in semi consistent state simulations and its consequences for the yielded and un-yielded areas. Gertzos et al. (2008) presented a new full CFD method in order to model the Bingham Plastic behavior and also the Bingham-Papanastasiou model in the lubricating film. The advantage of the technique is that it is furthermore suitable when the thin film approximation is not really valid. Kim et al. (2000) demonstrate the make use of a bi-viscous materials model implemented in a new CFD model to simulate the behavior in the Bingham plastic material in the lubricating film. Patel and chhabra, (2015) studied numerically the free convection flow from a good isothermal elliptical cylinder within Bingham plastic Fluids.

In modern engineering, many fluids show non -Newtonian behavior, therefore many researchers are more interested in those industrial non-Newtonian fluids and their dynamics (Banerjee et al., 2018). In many of the research problems lube is presumed to be Newtonian. Considering that the lubricant subject to heavy load, the Newtonian fluid properties usually tend not to be found for longer time. Therefore, the non-Newtonian qualities have to be offered in various lubrication issues. So the effects associated with non-Newtonian characteristics involving the lubricant are to be incorporated along with the pressure and temperature (Prasad and Subrahmanyam, 2014). Kim together with Seireg (2000) developed a new computational method for forecasting the dimension of the particular shear zone in thermo hydrodynamic lubrication by making use of Bingham model for the lubricant which is presumed to behave rheological pressure. Jang and Khonsari (2001) provided three dimensional solutions for the slider bearing lubes with Bingham grease using full thermo hydrodynamic thought. Salehizadeh and Saka (1991) attempted a problem on thermal effects related to pure rolling but not sliding of cylindrical roller bearings lubricated by re-eyring non-Newtonian fluids. The temperature dispersion over the film was expected so as to be parabolic and viscosity to be the function of the mean temperature. Prasad et al.

\footnotetext{
* Currently in Department of Mathematics, Gokaraju Rangaraju Institute of Engineering and Technology, Hyderabad, India.

† Corresponding author.Email: grevathi1331@gmail.com
} 
(2012) analyzed thermo hydrodynamic lubrication features involving asymmetric rollers lubricated by incompressible power-law fluids intended for highly packed rigid line contact system assuming the fluid consistency to be changed with pressure and mean film temperature. Further, it was concluded that considerable increase was observed in mean temperature with flow behavior index and rolling ratio. Sajja and Prasad (2015) studied effects of temperature in hydrodynamic lubrication of incompressible non-Newtonian power-law fluids intended for highly filled rigid system and concluded that a notable change was observed in the mean film temperature with flow index and rolling ratio. Ravi Datt et al. (2019) presented a critical review of the work performed in the area of hydrodynamic and thermal performance using twisted tapes. The effect of various twisted tape inserts parameters on hydrodynamic and thermal performance are discussed in detail.

In the light of the above literature, the purpose of the present paper is related to study of thermal effects in hydrodynamic lubrication of asymmetric rollers by an incompressible Bingham plastic fluid for highly supplied rigid system under the operational behavior of line contact presuming the fluid viscosity to differ with hydrodynamic pressure under isothermal boundaries. The rolling ratio is used to investigate the rolling and sliding effects of surfaces on the temperature, pressure, load and traction.

\section{THEORETICAL MODEL}

Lubrication system of asymmetric roller bearings for highly freighted rigid system by incompressible non-Newtonian Bingham plastic fluid is considered in this paper in such a way that both the surfaces are having same radius and moving with different velocities. Further it can be noted that the velocity of the lower surface is higher than the upper surface. The flow configuration is shown in Fig. 1.

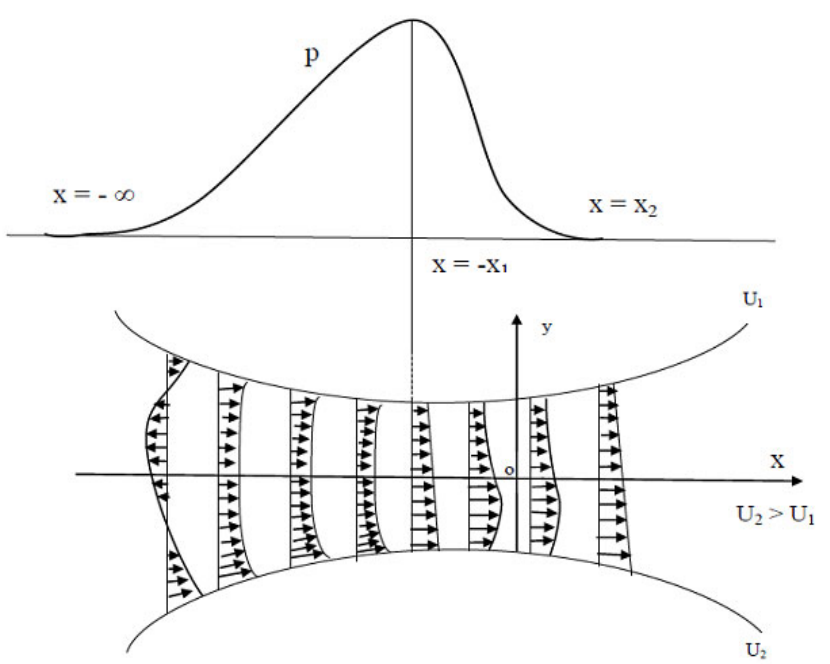

Fig. 1 Lubrication of Asymmetric Rollers

\subsection{Formulation of the Problem}

The equations such as continuity and momentum which govern the flow of an in-compressible fluid within the content of a typical lubrication problem are considered under usual assumptions (Prasad et al., 1987) are as follows:

$$
\begin{aligned}
& \frac{\partial u}{\partial x}+\frac{\partial v}{\partial y}=0 \\
& \frac{d p}{d x}=\frac{\partial \tau}{\partial y}
\end{aligned}
$$

where ' $\mathrm{p}$ ' and ' $\tau$ ' are hydrodynamic pressure and shear stress respectively. The constitutive relation for Bingham plastic fluid is given by (Tokio Sasaki et al., 1962)

$$
\tau= \pm \tau_{0}+\mu \frac{\partial u}{\partial y}
$$

where $\mu$ is the viscosity of the fluid taken by

$$
\mu=\mu_{0} e^{\alpha p}
$$

and the equation for thickness of the film is to be $h=h_{0}+\frac{x^{2}}{2 R}$

\subsection{The boundary conditions}

The boundary conditions for this problem at both upper and lowers surfaces are taken as

$$
\begin{aligned}
& u=U_{1} \text { at } y=h \\
& u=U_{2} \text { at } y=-h \\
& p=0 \text { at } x=-\infty ; p=0 \text { and } p^{\prime}(x)=0 \text { at } x=x_{2}
\end{aligned}
$$

where $U_{1}$ and $U_{2}$ are velocities of the rolling cylinders as shown in Fig.(1). The expression for the velocity of the fluid can be obtained by solving the equation (2) using the boundary conditions mentioned above as given below

$$
\begin{aligned}
& u=\frac{3}{4 h^{3}}\left(U_{1}+U_{2}\right)\left(h-h_{1}\right)\left(y^{2}-h^{2}\right)+\frac{y}{2 h}\left(U_{1}-U_{2}\right)+\frac{1}{2}\left(U_{1}+U_{2}\right) \\
& Q=\int_{-h}^{h} u d y=h\left(U_{1}+U_{2}\right)-\frac{2 h^{3}}{3 \mu} \frac{d p}{d x}
\end{aligned}
$$

And the volume flux at the point of maximum pressure is $Q\left(-x_{1}\right)=\left(U_{1}+U_{2}\right) h_{1}$

Where the film thickness $h_{1}$ at $x=-x_{1}$ is regarded to be $h_{1}=1+x_{1}{ }^{2}$

\subsection{Reynolds equation}

Integrating equation (2) using the boundary conditions (eqn. 5-7) gives the pressure Reynolds equation which is presented below

$\frac{d p}{d x}=\frac{3 \mu}{2 h^{3}}\left(U_{1}+U_{2}\right)\left(h-h_{1}\right)$

\subsection{Dimensionless scheme}

The following dimensionless scheme for roller bearings is applied throughout this paper

$$
\begin{aligned}
& \bar{x}=\frac{x}{R}, \quad \bar{p}=\alpha p, \quad \overline{\mathrm{h}}=1+\bar{x}^{2}, \bar{\mu}=\overline{\mu_{0}} e^{\bar{p}}, \\
& \bar{\delta}=\frac{\delta}{h_{0}}, \overline{\mu_{0}}=\frac{2 \alpha R U}{h_{0}{ }^{2}} \mu_{0}, \overline{\mathrm{U}}=\frac{U_{2}}{U_{1}}, \overline{\mathrm{T}_{\mathrm{m}}}=\beta T_{m}
\end{aligned}
$$

The velocity expression and pressure Reynolds equation are written in dimensionless form using the above mentioned dimensionless scheme.

$$
\begin{aligned}
& \bar{u}=\frac{3}{4 \bar{h}^{3}}(1+\bar{U})\left(\bar{h}-\overline{h_{1}}\right)\left(\bar{y}^{2}-\bar{h}^{2}\right)+\frac{\bar{y}}{2 \bar{h}}(1-\bar{U})+\frac{1}{2}(1+\bar{U}) \\
& \frac{d \bar{p}}{d \bar{x}}=\frac{3 \bar{\mu}(1+\bar{U})\left(\bar{h}-\overline{h_{1}}\right)}{4 \bar{h}^{3}}
\end{aligned}
$$

\subsection{Heat Equation:}

Let us assume the following heat equation for the line contact lubrication problem (Sajja and Prasad, 2015)

$k \frac{\partial^{2} T}{\partial y^{2}}+\tau \frac{\partial u}{\partial y}=0$

where the shear stress $\tau$ for Bingham plastic fluid is taken from equation (2a).

The following are the boundary conditions for the above heat equation $T=T_{U}$ at $y=h$ and $T=T_{L}$ at $y=-h$

Where $T_{U}$ and $T_{L}$ are the temperatures of the top and lower surfaces respectively. Integration of equation (14) gives the temperature of the lubricant as given below: 


$$
\begin{aligned}
T= & \left(\frac{T_{U}+T_{L}}{2}\right)-\frac{\tau_{0}}{k}\left[\frac{y^{3}}{4 h^{3}}\left(U_{1}+U_{2}\right)\left(h-h_{1}\right)+\frac{\left(U_{1}-U_{2}\right) y^{2}}{4 h}\right] \\
& -\frac{\mu}{k}\left[\frac{3 y^{4}\left(U_{1}+U_{2}\right)^{2}\left(h-h_{1}\right)^{2}}{16 h^{6}}+\frac{\left(U_{1}-U_{2}\right)^{2} y^{2}}{8 h^{2}}+\frac{y^{3}\left(U_{1}{ }^{2}-U_{2}{ }^{2}\right)\left(h-h_{1}\right)}{4 h^{4}}\right] \\
& +y\left[\frac{T_{U}-T_{L}}{2 h}+\frac{\tau_{0}}{4 k h}\left(U_{1}+U_{2}\right)\left(h-h_{1}\right)+\frac{\mu\left(U_{1}{ }^{2}-U_{2}{ }^{2}\right)\left(h-h_{1}\right)}{4 k h^{2}}\right]+ \\
& +\frac{\tau_{0} h}{4 k}\left(U_{1}-U_{2}\right)+\frac{\mu}{2 k}\left[\frac{3\left(U_{1}+U_{2}\right)^{2}\left(h-h_{1}\right)^{2}}{8 h^{2}}+\frac{\left(U_{1}-U_{2}\right)^{2}}{4}\right]
\end{aligned}
$$

Thus, the temperature $\mathrm{T}$ is analytically known function of $\mathrm{x}$ and $\mathrm{y}$ explicitly. Now, the temperature $T_{m}$ in average form is given by $T_{m}=\int_{-h}^{h} T d y$ and can be written as:

$T_{m}=\frac{T_{U}+T_{L}}{2}+\frac{\tau_{0} h}{6 k}\left(U_{1}-U_{2}\right)+\frac{\mu}{4 k h}\left[\frac{3\left(U_{1}+U_{2}\right)^{2}\left(h-h_{1}\right)^{2}}{5 h}+\frac{\left(U_{1}-U_{2}\right)^{2} h}{3}\right]$

Now the dimensionless temperature and mean temperatures are obtained as follows

$$
\begin{aligned}
\bar{T} & =\frac{\bar{h}\left(\overline{T_{U}}+\overline{T_{L}}\right)+\bar{y}\left(\overline{T_{U}}-\overline{T_{L}}\right)}{2 \bar{h}}+\bar{\lambda}\left[\frac{\left(1-\bar{U}\left(\bar{h}^{2}-y^{2}\right)\right.}{\bar{h}}+\frac{(1+\bar{U})\left(\bar{h}-\overline{h_{1}}\right)\left(\bar{h}^{2}-y^{2}\right) \bar{y}}{\bar{h}^{3}}\right] \\
& +\bar{\gamma} e^{p}\left[\frac{(1-\bar{U})^{2}\left(\bar{h}^{2}-\bar{y}^{2}\right)}{2 \bar{h}^{2}}+\frac{3(1+\bar{U})^{2}\left(\bar{h}-\bar{h}_{1}\right)^{2}\left(\bar{h}^{4}-\bar{y}^{-4}\right)}{\bar{h}^{6}}+\frac{\left(1-\bar{U}^{2}\right)\left(\bar{h}-\overline{h_{1}}\right)\left(\bar{h}^{2}-\bar{y}^{2}\right) \bar{y}}{\bar{h}^{4}}\right] \\
\overline{T_{m}} & =\frac{\left(\overline{T_{U}}+\overline{T_{L}}\right)}{2}+\frac{2 \bar{h} \bar{\lambda}}{3}(1-\bar{U})+\frac{\overline{\gamma e} \bar{e}^{p}}{\bar{h}}\left[\frac{3(1+\bar{U})^{2}\left(\bar{h}-\overline{h_{1}}\right)^{2}}{5 \bar{h}}+\frac{(1-\bar{U})^{2} \bar{h}}{3}\right]
\end{aligned}
$$

\subsection{Load and Traction:}

The load capacity is one of the important characteristics of loaded bearings because it provides an overall estimate of the efficiency of the bearings. Hence its calculation is very essential. Integration of the pressure across the film thickness gives the x-component of the load $W_{x}$ per unit length of the cylinder as

$W_{x}=\int_{-h}^{h} p d h$

The non-dimensional load $\bar{W}=\frac{w \alpha}{R}$ is given by

$\overline{W_{x}}=\int_{-\infty}^{\bar{x}_{2}} \bar{x} \frac{d \bar{p}}{d \bar{x}} d \bar{x}$

Similarly, the y-component $W_{y}$ of the load is obtained as

$\overline{W_{y}}=-\int_{-\infty}^{\bar{x}_{2}} \bar{x} \frac{d \bar{p}}{d \bar{x}} d \bar{x}$

Further, the load components $\mathrm{W}$ is determined by

$W=\sqrt{W_{x}^{2}+W_{y}^{2}}$

Now, the traction force $T_{F}$ at the surfaces can be acquired by solving the shear stress $\tau$ for the whole length as

$T_{F h-}=-\int_{-\infty}^{x_{2}} \tau_{y=-h} d x ;$ and $T_{F h+}=-\int_{-\infty}^{x_{2}} \tau_{y=h} d x$

Dimensionless tractions are

$\bar{T}_{F h-}=-\int_{-\infty}^{\bar{x}_{2}} \bar{\tau}_{\bar{y}=-\bar{h}} d \bar{x} ; \quad$ and $\bar{T}_{F h+}=-\int_{-\infty}^{\bar{x}_{2}} \bar{\tau}_{\bar{y}=\bar{h}} d \bar{x}$

\section{RESULTS AND DISCUSSION}

Numerical computations are performed with the following values in this problem:

$U_{2}=400 \mathrm{~cm} / \mathrm{s}, h_{0}=4 \times 10^{-4} \mathrm{~cm}, \alpha=1.6 \times 10^{-9} \mathrm{dyne}^{-1} \mathrm{~cm}^{2}, R=3 \mathrm{~cm}$,

$\bar{\gamma}=10, \overline{T_{L}}=1, \overline{T_{U}}=0.5$

\subsection{Pressure profile}

The pressure $\bar{p}$ distributions are depicted in Fig. 2 and can be observed that the lubricant pressure $\bar{p}$ increases with rolling ratio $\bar{U}$.

This indicates that hydrodynamic pressure is more for sliding case than pure rolling. The trend similar to this behavior was observed in Revathi et al. (2019); Prasad et al. (2012); Sajja and Prasad (2015); Prasad and Sajja (2016); Prasad and Sajja (2016a). Further, the points of maximum pressure with respect to rolling/ sliding ratio $\bar{U}$ are presented in the Table 1. This gives the information that point of maximum pressure shift towards the central point of contact as rolling/ sliding ratio increases.

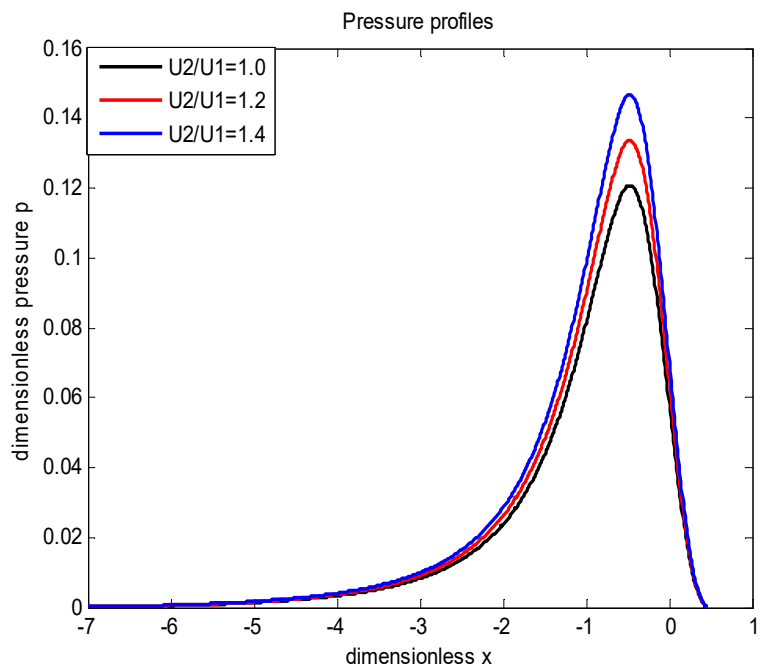

Fig. 2 Pressure profile for different $\bar{U}$

Table 1 Points of maximum pressure

\begin{tabular}{|c|c|}
\hline $\bar{U}$ & $\bar{x}_{1}$ \\
\hline 1.0 & 0.47390268209725 \\
\hline 1.1 & 0.47390264253725 \\
\hline 1.2 & 0.47390260293489 \\
\hline 1.3 & 0.47390256331725 \\
\hline 1.4 & 0.47390252363725 \\
\hline 1.5 & 0.47390248403725 \\
\hline
\end{tabular}

\subsection{Velocity profile}

The fluid velocity $\bar{u}$ profiles are numerically computed at different locations and presented in Fig. 3, Fig. 4 and Fig. 5 for the zones before, after, and also at the point of maximum pressure. They appear in parabolic shapes with the vertices in downward and upward directions in the zones before and after the point of maximum pressure. The vertices below the $\bar{y}$ line show there is a back flow near the inlet as presented in Fig. 3. The flow in reverse direction was also presented by Dong Zhu and Wen, 1984. As the fluid progresses, the back flow is over. The same is also shown roughly in Fig. 1, Prasad et al., (1988), Prasad et al., (1991).

Further, the velocity profile, at the point of maximum pressure, seems to be linearly decreasing and can be observed in Fig. 5. It is because pressure gradient at that point is zero. This shows that pressure is maximum there. 


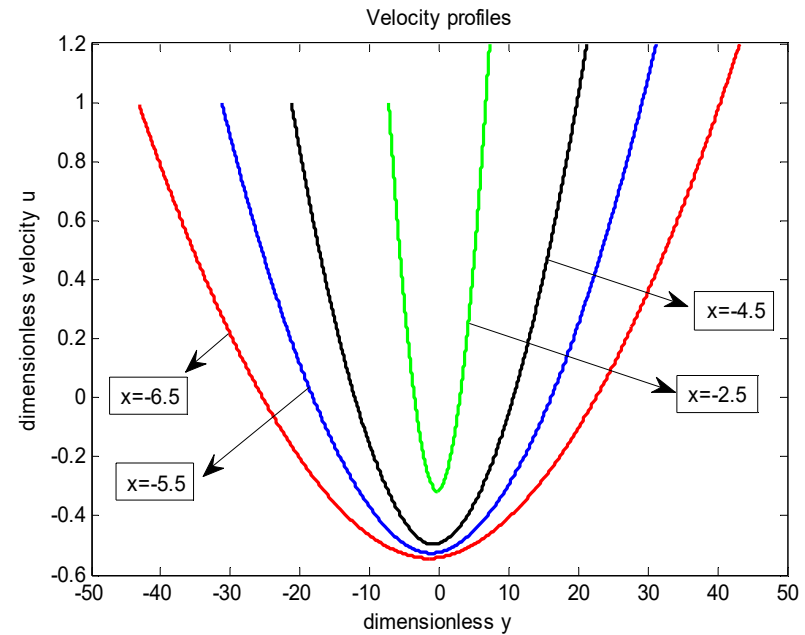

Fig. 3 Velocity Profile

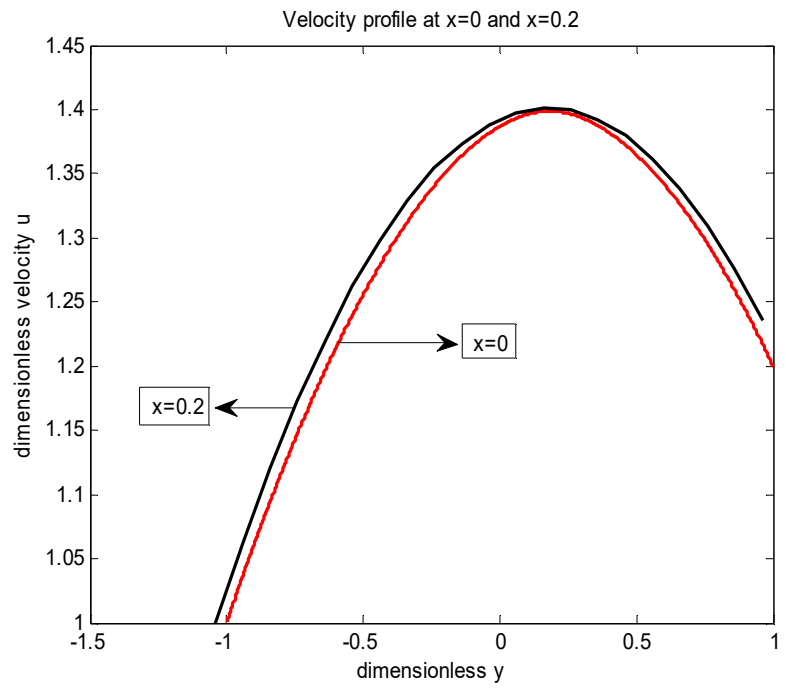

Fig. 4 Velocity profile

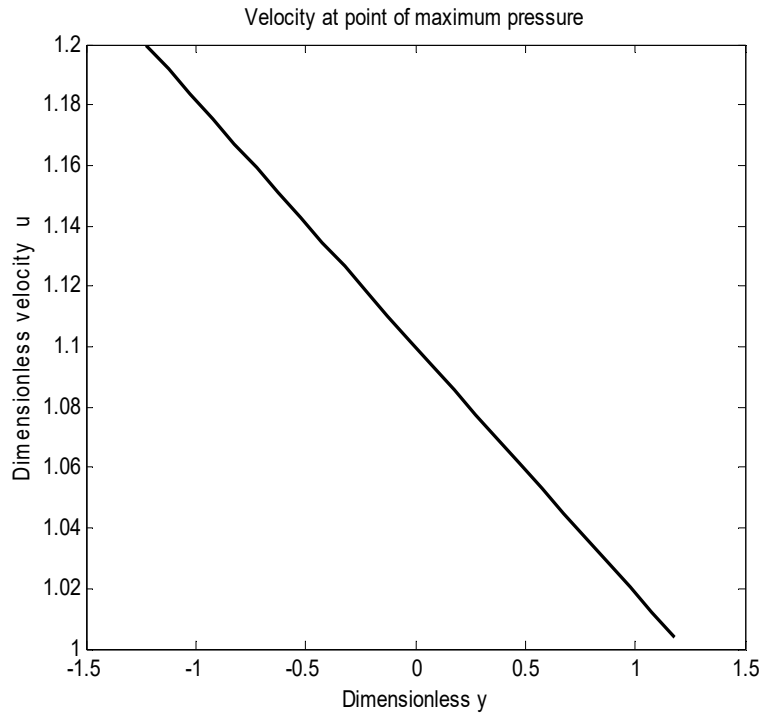

Fig. 5 Velocity profile at point of maximum pressure

\subsection{Temperature Profiles}

The dimensionless temperature $\bar{T}$ of the lubricant is computed numerically and presented in the form of the following graphs (Fig. 6- Fig. 7) for various values of rolling ratio $\bar{U}$ and $\bar{\tau}_{0}$. The dimensionless temperature profile for pure rolling case $\left(\bar{U}=U_{2} / U_{1}=1\right)$ with $\bar{\tau}_{0}=0$ (Newtonian case) was presented in Fig. 6 . The dimensionless temperature profile for sliding case $(\bar{U}=1.2)$ with $\bar{\tau}_{0}=1$ (Non-Newtonian case) was presented in Fig. 7. The numerical computations for other values of the parameters are also done and not given here since they are qualitatively very similar to the figures presented in Fig. 6 and Fig. 7.

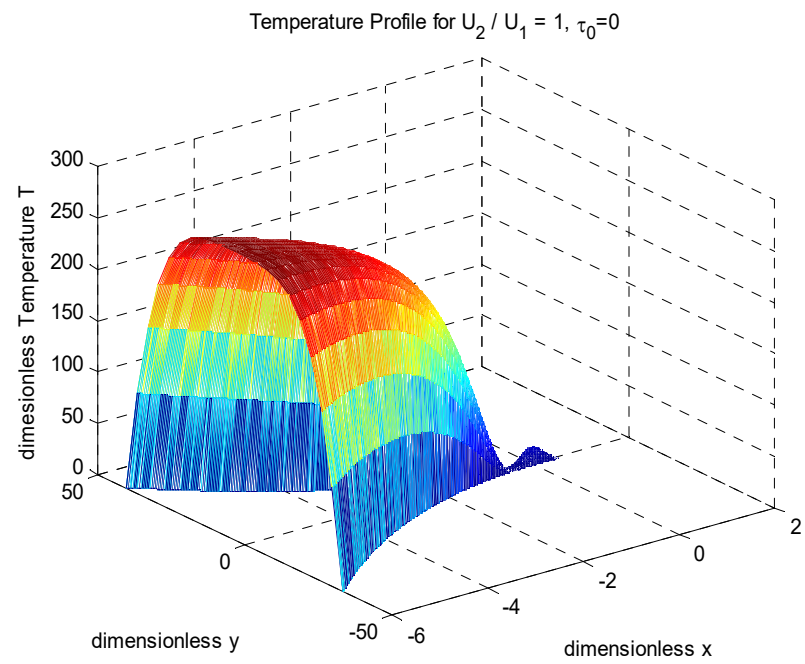

Fig. 6 Temperature $\bar{T}$ profile

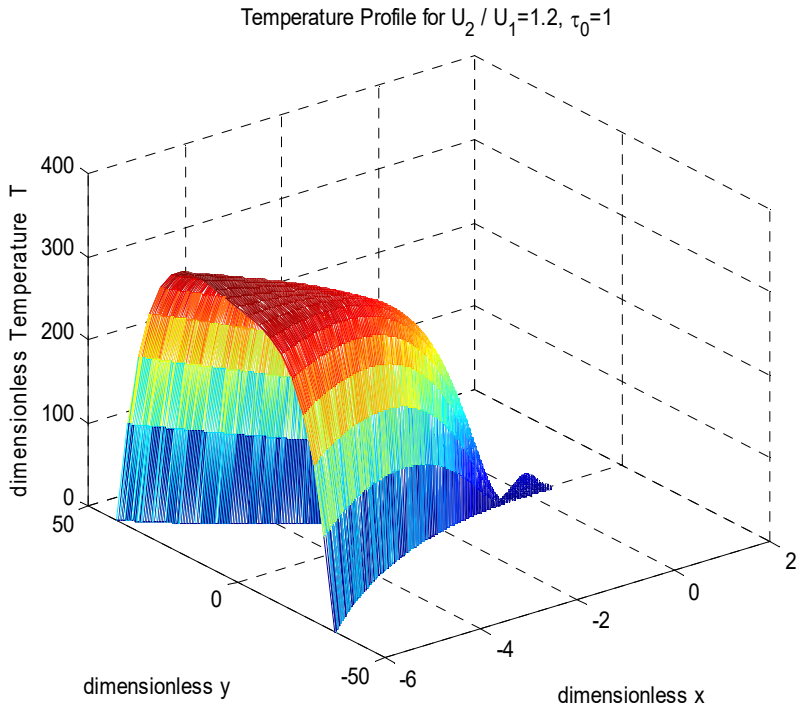

Fig. 7 Temperature $\bar{T}$ profile

\subsubsection{Mean Temperature profiles}

The dimensionless mean temperature $\bar{T}_{m}$ of the lubricant is elaborated in Fig. 8 - Fig. 11 at various values of rolling ratio $\bar{U}$ and $\bar{\tau}_{0}$. The dimensionless mean temperature profile for pure rolling case $\bar{U}=1$ with different values of $\bar{\tau}_{0}$ is presented in Fig. 8 and can be observed that $\bar{T}_{m}$ coincides with different values of $\bar{\tau}_{0}$. The mean temperature $\bar{T}_{m}$ is presented in Fig. 9 for fixed values of sliding parameter $\bar{U}=1.2$ and different values of $\bar{\tau}_{0}$. One can see from this figure that mean temperature decreases with increase of $\bar{\tau}_{0}$. Fig. 10 and Fig. 11 show 
that the mean temperature enhances with $\bar{U}$ for fixed value of $\bar{\tau}_{0}$ and indicates that the mean temperature for sliding case is higher when compared to that of pure rolling (Sajja and Prasad, 2015; Prasad and Sajja, 2016; Prasad and Sajja , 2016a). Further, in all the Temperature profiles, $\bar{T}_{m}$ decreases throughout the inlet region up to the point of maximum pressure $\bar{x}=-\bar{x}_{1}$, then it increases up to the origin and thereafter decreases up to the cavitation point $\bar{x}=\bar{x}_{2}$. However, an increase or a decrease in $\bar{T}_{m}$ beyond the point $\bar{x}=-\bar{x}_{1}$ is hardly discernible. The reason for the shape of the curves in the Fig. 8- Fig. 11 is that, the temperature goes down in inlet region because of heat lost due to conduction as flow proceeds. It may also be observed that the subjective behaviors of $\bar{T}_{m}$ versus $\bar{x}$ are very indistinguishable to the temperature profile obtained by Prasad et al., 1991; Saini et al., 2007 for the case of Power-law fluids and symmetric case.

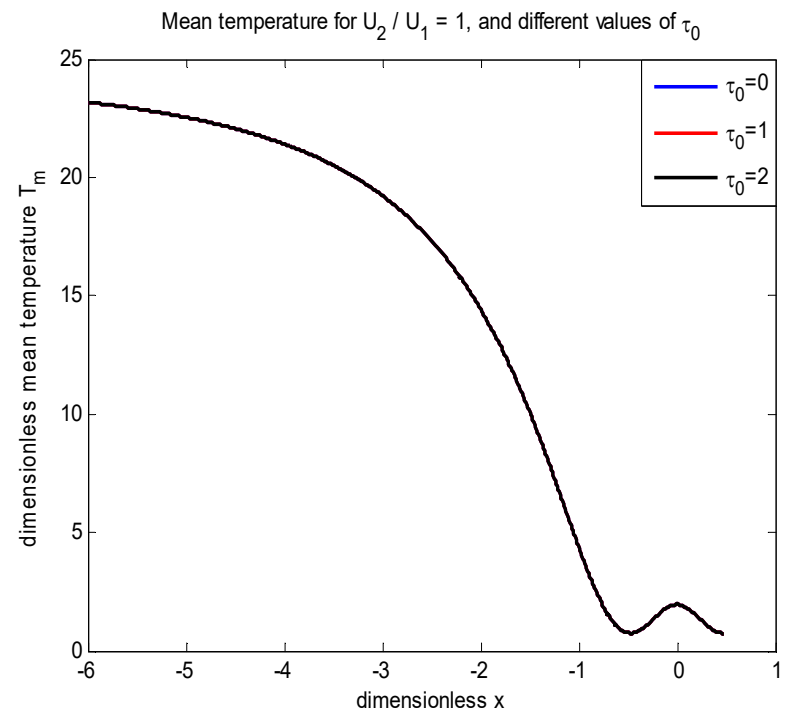

Fig. 8 Mean Temperature $\bar{T}_{m}$ profile

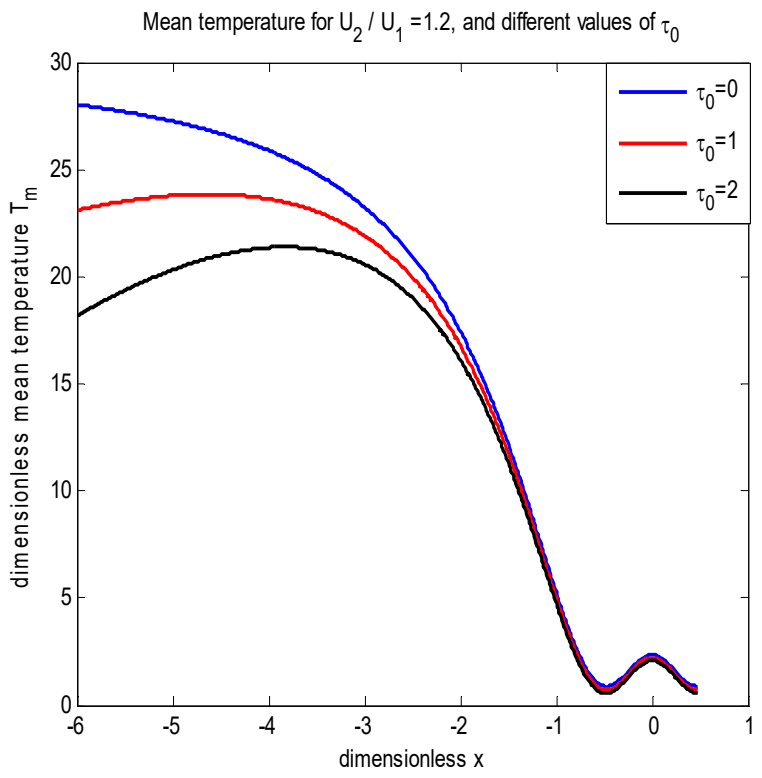

Fig. 9 Mean Temperature $\bar{T}_{m}$ profile

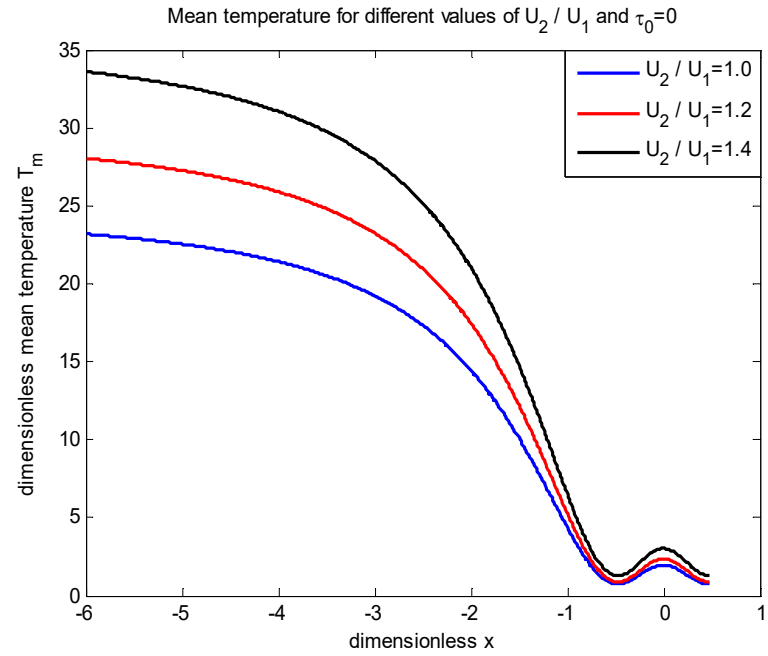

Fig. 10 Mean Temperature $\bar{T}_{m}$ profile

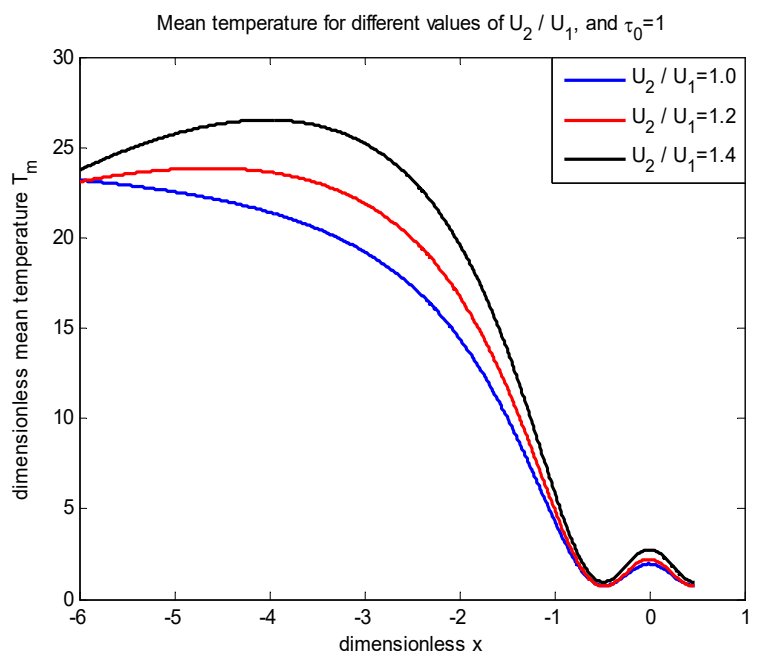

Fig. 11 Mean Temperature $\bar{T}_{m}$ profile

\subsection{Viscosity $\left({ }_{\mu}\right)$ Profile}

The lubricant viscosity $\bar{\mu}_{\mu}$ is calculated numerically and shown in Fig. 12. One can observe from the figure that the lubricant viscosity profile is similar to pressure profile. Since the viscosity considered in this problem is a function of hydrodynamic pressure, hence, the viscosity profile is similar to pressure profile. The same trend was observed when Power-law fluid considered by Sajja and Prasad (2015).

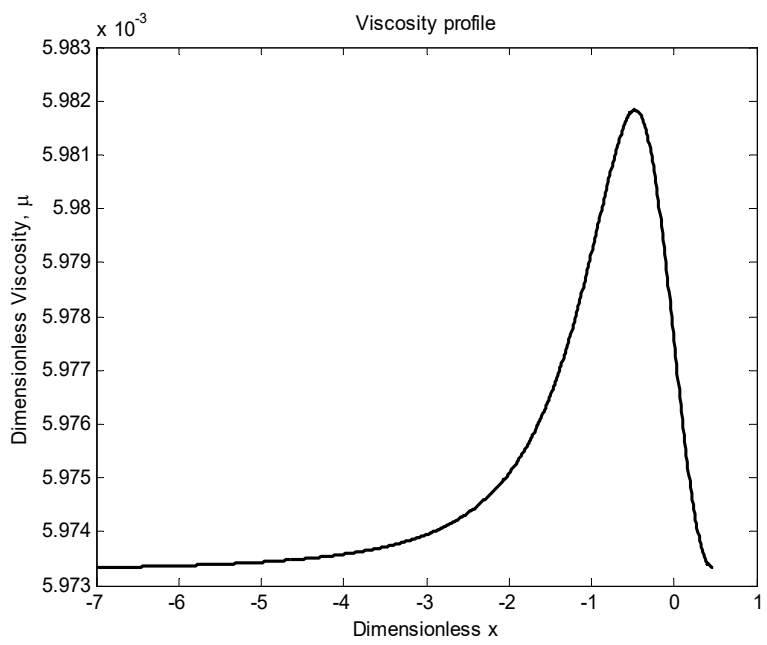

Fig. 12 Viscosity ${ }_{\mu}$ profile 


\subsection{Load and Traction}

Numerically computed values of the dimensionless load $\overline{W_{x}}$ in $\mathrm{x}$-direction are presented graphically in Fig. 13 and it can be seen that $\overline{W_{x}}$ increases with rolling ratio $\bar{U}$. Further, normal load carrying capacity $\overline{W_{y}}$ in dimensionless form in y-direction is presented in Fig.

14. The trends of load in both the profiles are increasing with rolling ratio and have the same characteristics. It is in conformity with the previous findings of Salehizadeh and Saka (1991); Prasad et al. (2012); Sajja and Prasad (2015); Prasad et al. (1987) for Power-law case for both the symmetric and asymmetric cases.

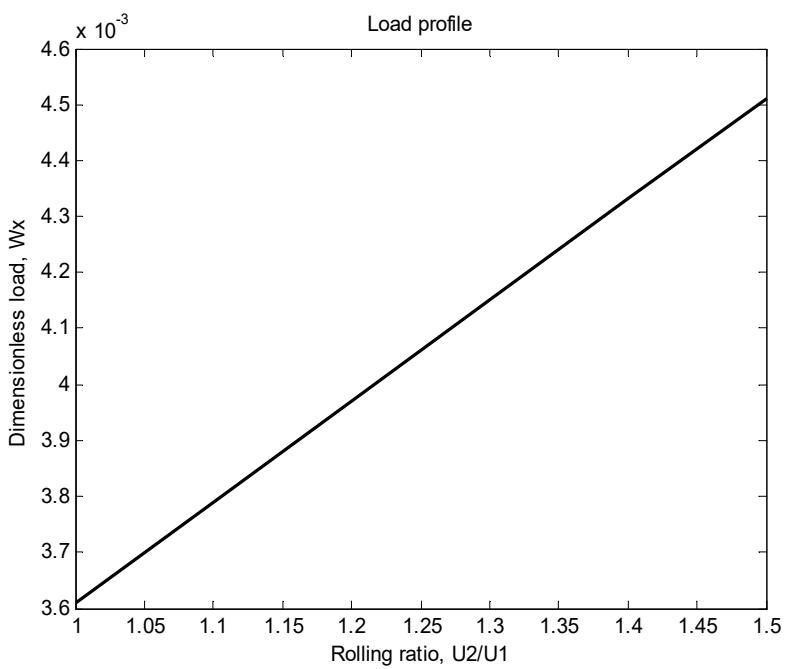

Fig. 13: Load $\overline{W_{x}}$ in $\mathrm{x}$-direction

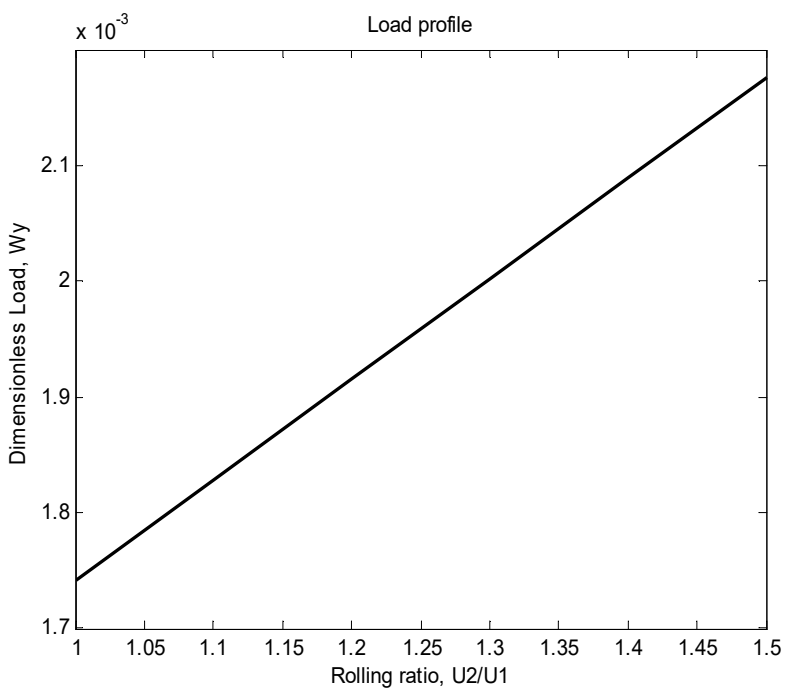

Fig. 14: Load $\overline{W_{y}}$ in y-direction

The traction forces $\bar{T}_{F h}$ have been evaluated and presented in the tables 1 and 2 at both the surfaces for distinct values of $\bar{\tau}_{0}$ and $\bar{U}$. Here $\bar{\tau}_{0}=0$ represents Newtonian case and $\bar{\tau}_{0}>0$ represents non-Newtonian case. The Traction forces increase at both the surfaces with $\bar{\tau}_{0}$ for fixed value of $\bar{U}$. Further, the increase of traction forces with $\bar{U}$ at lower surface can be observed from table 1 this is because that the lower surface moving with more velocity than upper surface and gets more traction force. This trend was observed for Power-law fluid case by in Prasad et al. (2012); Sajja and Prasad (2015); Prasad and Sajja (2016). Both bearings experience the same traction force when $\bar{U}=1$.
Table 2 Traction values at lower surface

\begin{tabular}{|l|l|l|l|}
\hline $\bar{U}$ & when $\bar{\tau}_{0}=0$ & when $\bar{\tau}_{0}=0.5$ & when $\bar{\tau}_{0}=1$ \\
\hline 1.0 & 0.00361232 & 2.79361232 & 5.58361232 \\
\hline 1.1 & 0.00407083 & 2.79407083 & 5.58407083 \\
\hline 1.2 & 0.00452935 & 2.79452935 & 5.58452935 \\
\hline 1.3 & 0.00498789 & 2.79498789 & 5.58498789 \\
\hline 1.4 & 0.00544643 & 2.79544643 & 5.58544643 \\
\hline 1.5 & 0.00590499 & 2.79590499 & 5.58590499 \\
\hline
\end{tabular}

Table 3: Traction values at upper surface

\begin{tabular}{|c|c|c|c|}
\hline $\bar{U}$ & when $\bar{\tau}_{0}=0$ & when $\bar{\tau}_{0}=0.5$ & when $\bar{\tau}_{0}=1$ \\
\hline 1.0 & 0.00361232 & 2.79361232 & 5.58361232 \\
\hline 1.1 & 0.00351528 & 2.79351528 & 5.58351528 \\
\hline 1.2 & 0.00341824 & 2.79341824 & 5.58341824 \\
\hline 1.3 & 0.00332121 & 2.79332121 & 5.58332121 \\
\hline 1.4 & 0.00322420 & 2.79322420 & 5.58322420 \\
\hline 1.5 & 0.00312719 & 2.79312719 & 5.58312719 \\
\hline
\end{tabular}

\section{CONCLUSIONS}

The problem is devoted to study the thermal lubrication of rolling /sliding line contact by an incompressible Bingham plastic fluid under the assumption of isothermal boundaries. The pressure and the thermal energy equation are solved for pressure and the mean temperature simultaneously for various values of $\bar{\tau}_{0}$ and the sliding parameter $\bar{U}$.

The following facts may be observed from the results of the work here:

(i) Both the velocity and pressure of the lubricant are independent of $\bar{\tau}_{0}$

(ii) There is a notable increase in pressure with respect to rolling ratio $\bar{U}$.

(iii) Velocity of the lubricant at point of maximum pressure decreases linearly.

(iv) Load in both $\mathrm{x}$ and $\mathrm{y}$ directions increases with rolling ratio $\bar{U}$.

(v) The traction at the lower surface is higher than that of the upper surface because of the more speed of the lower surface.

(vi) Traction forces increase at both the surfaces with $\bar{\tau}_{0}$ for fixed $\bar{U}$

(vii) Mean temperature decreases as $\bar{\tau}_{0}$ increases.

(viii) Temperature in sliding case is more than the case of pure rolling. (ix) A significant enhancement in mean film temperature is observed with respect to $\bar{U}$.

\section{NOMENCLATURE}

P Hydrodynamic pressure

$\mathrm{R} \quad$ Radius of the equivalent cylinder

$\mathrm{T} \quad$ Lubricant temperature

$\mathrm{T}_{0} \quad$ Ambient temperature

$\mathrm{T}_{\mathrm{Fh}} \quad$ Traction force

$\mathrm{u} \quad$ Velocity of the lubricant in $\mathrm{x}$-direction

$\mathrm{v} \quad$ Velocity of the lubricant in y-direction

$\mathrm{x}_{1} \quad$ Point of maximum pressure

$\mathrm{x}_{2} \quad$ Cavitation point

$\mathrm{k} \quad$ Thermal conductivity of the lubricant

h Film thickness

ho Minimum film thickness

a Pressure coefficient

$\mathrm{U}_{1}, \mathrm{U}_{2} \quad$ Velocities of the surfaces 


\section{REFERENCES}

Banerjee, A., Zaib, A., Bhattacharyya, K., Mahato, S.K., 2018, "MHD mixed convection flow of a non-Newtonian Powell-Erying fluid over a permeable exponentially shrinking sheet," Frontiers in Heat and Mass Transfer, 10(30), 1-8.

https://doi.org/10.5098/hmt.10.30

Dorier, C., and Tichy, J., 1992, "Behavior of Bingham-like viscous fluid in lubrication flows," Journal of Non-Newtonian Fluid Mechanics, 45, 291-310.

https://doi.org/10.1016/0377-0257(92)80065-6

Prasad, D., and Subrahmanyam, S.V., 2014, "Thermo hydrodynamic lubrication characteristics of power law fluids in Rolling sliding line contact," Proceedings of International Conference and Advances in Tribology and Engineering Systems, Lecture Notes in Mechanical Engineering, springer India,

https://doi.org/10.1007/978-81-322-1656-8_11

Prasad, D., Singh, P., and Sinha, P., 1987, "Thermal and squeezing effects in non-Newtonian fluid film lubrication of rollers," Wear, 119, 175-190.

https://doi.org/10.1016/0043-1648(87)90107-4

Prasad, D., Sajja, V.S., 2016, "Non-Newtonian lubrication of asymmetric rollers with thermal and inertia effects," Tribology Transactions, 59(5), 818-830.

https://doi.org/10.1080/10402004.2015.1107927

Prasad, D., Sajja, V.S., 2016, "Thermal effects in non-Newtonian lubrication of asymmetric rollers under adiabatic and isothermal boundaries," International Journal of Chemical Sciences, 14(3), 16411656.

Zhu, D., and Wen, S.Z., 1984, "A full numerical solution for the thermal elastohydrodynamic problem in elliptical contacts," Journal of Tribology, 106, 246-254.

https://doi.org/10.1115/1.3260895

Revathi, G., Sajja, V.K., and Prasad, D. 2019, "Thermal effects in Power-law fluid film lubrication of rolling/sliding line contact," International Journal of Innovative Technology and Exploring Engineering, 2019, 8(8), 277-283.

https://doi.org/10.35940/ijitee.H7195.078919

Gertzos, K. P., Nikolakopoulos, P.G., Papadopoulos, C. A., 2008, "CFD Analysis of journal bearing hydrodynamic lubrication by Bingham lubricant," Tribology International, 41(12), 1190-204.

https://doi.org/10.1016/j.triboint.2008.03.002

Jang, J.Y., Khonsari, M.M., 2001, "On thermohydrodynamic analysis of a Bingham fluid in slider bearings," Acta Mechanica, 148, 165-185. https://doi.org/10.1007/BF01183676

Kim, J. H., Seireg, A. A., 2000, "Thermo hydrodynamic lubrication analysis incorporating Bingham rheological model," Journal of Tribology, 122, 137-146.

https://doi.org/10.1115/1.555336

Kim, P., Lee, J. I, Seok, J., 2014, “Analysis of a viscoplastic flow with field-dependent yield stress and wall slip boundary conditions for a magnetorheological (MR) fluid". Journal of Non-Newtonian Fluid Mechanics, 204, 72-86. https://doi.org/10.1016/j.jnnfm.2013.12.005

Milne, A. A., 1954, “A Theory of rheodynamic lubrication,” Kolloid-Z, 139, 96-101.
Mitsoulis, E., and Matsoukas, A., 2005, "Free surface effects in squeeze flow of Bingham plastics," Journal of Non-Newtonian Fluid Mechanics, 129, 182-187.

https://doi.org/10.1016/j.jnnfm.2005.06.002

Patel, S.A., Chhabra, R. K., 2016, "Laminar free convection in Bingham plastic fluids from an isothermal elliptical cylinder," Journal of Thermophysics and Heat Transfer, 30(1), 152-167.

https://doi.org/10.2514/1.T4578

Prasad D., Shukla J.B., Singh P., Sinha P., Chhabra R.P., 1991, "Thermal effects in lubrication of asymmetrical rollers," Tribology International, 24, 239-246.

https://doi.org/10.1016/0301-679X(91)90050-J

Prasad D., Singh P., Sinha P., 1988, "Non-uniform temperature in nonNewtonian compressible fluid film lubrication of rollers," Journal of Tribology, 110, 653-658.

https://doi.org/10.1115/1.3261708

Prasad, D., Subrahmanyam, S.V., Sudam Sekhar Panda, 2012, "Thermal effects in hydrodynamic lubrication of asymmetric rollers using R-K Fehlberg method," International Journal of Engineering Science and Advanced Technology, 2(3), 422-437.

Sinha, P., and Singh, C., 1982, "Lubrication of a cylinder on a plane with a non-Newtonian fluid considering cavitation," Journal of Lubrication Technology, 104, 168-172.

https://doi.org/10.1115/1.3253176

Datt, R., Kumar, A., Bhist, M.S., Kothiyal, A.D., Maithani, R., 2019, "Hydrodynamic and thermal performance of twisted tape insert provided in heat exchanger tubes: a review," Frontiers in Heat and Mass Transfer, 12(26), 1-19.

https://doi.org/10.5098/hmt.12.26

Saini, P.K., Kumar, P and Tandon, P., 2007, "Thermal elastohydrodynamic lubrication characteristics of couple stress fluids in rolling/sliding line contacts," Journal of Engineering Tribology, 221, 141-153. https://doi.org/10.1243/13506501JET212

Salehizadeh, H., Saka. N., 1991, "Thermal non-Newtonian EHD lubrication of rolling line contacts," Journal of Tribology, 113, 481491.

https://doi.org/10.1115/1.2920649

Stefan G.E. Lampert, Ron A.J. Van Ostayen, 2020, "Lubrication theory for Bingham plastic," Tribology International, 147, 106160. https://doi.org/10.1016/j.triboint.2020.106160

Subrahmanyam, S. V., Prasad, D., 2015, "Characterization of lubrication of asymmetric rollers including Thermal Effects," Industrial Lubrication and Tribology, 67(3), 246-255. https://doi.org/10.1108/ILT-04-2013-0048

Sasaki, T., Mori, H., Okino, N., 1962, "Fluid lubrication theory of roller bearing-Part I:Fluid lubrication theory for two rotating cylinders in contact," Journal of Basic Engineering, 84(1), 166-174. https://doi.org/10.1115/1.3657240

Vola, D., Boscardin, L., Latche, J.C., 2003, "Laminar unsteady flows of Bingham fluids: a numerical strategy and some benchmark results," Journal of Computational Physics, 187, 441-456. https://doi.org/10.1016/S0021-9991(03)00118-9 